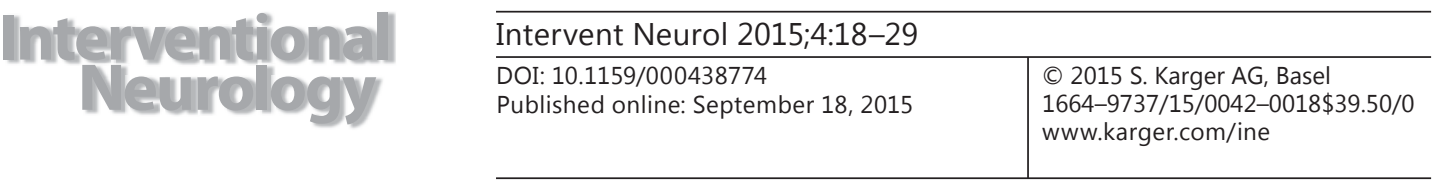

\title{
Mechanical Thrombectomy Is Now the Gold Standard for Acute Ischemic Stroke: Implications for Routine Clinical Practice
}

\author{
Murugan Palaniswami Bernard Yan \\ Melbourne Brain Centre, Royal Melbourne Hospital, University of Melbourne, Parkville, Vic., \\ Australia
}

\section{Key Words}

Mechanical thrombectomy · Neuroimaging $\cdot$ Recanalization - Stent retrievers - Stroke ·

Thrombolysis

\begin{abstract}
Background: This review aims to summarize the findings of the recently published randomized controlled studies which provide overwhelming evidence in support of mechanical thrombectomy for acute ischemic stroke with large artery occlusion. The five studies, Multicenter Randomized Clinical Trial of Endovascular Treatment for Acute Ischemic Stroke in the Netherlands (MR CLEAN), Endovascular Revascularization with Solitaire Device versus Best Medical Therapy in Anterior Circulation Stroke within $8 \mathrm{~h}$ (REVASCAT), Endovascular Treatment for Small Core and Proximal Occlusion Ischemic Stroke (ESCAPE), Solitaire ${ }^{\mathrm{TM}}$ FR as Primary Treatment for Acute Ischemic Stroke (SWIFT PRIME) and Extending the Time for Thrombolysis in Emergency Neurological Deficits with Intra-Arterial Therapy (EXTEND IA) have demonstrated the critical role of selecting patients by advanced neuroimaging, the superior recanalization capacity of stent retrievers and the effects of minimization of work processes delay. Summary: This review outlines lessons gained from the 5 positive studies which assessed mechanical thrombectomy as part of endovascular therapy for patients with proximal artery occlusion in the internal carotid and middle cerebral arteries. It discusses the role of age and stroke severity on treatment while also comparing the unique trial designs and selection criteria used amongst the 5 studies. In addition to examining the importance of unique imaging parameters such as collateral circulation, mismatch ratio and ischemic core volume, the review outlines differences in workflow parameters within the context of outcome. Finally the ben-
\end{abstract}

The content of this paper was presented at the 11th International Stroke Summit, Nanjing, 2015.

Bernard Yan

Melbourne Brain Centre

Royal Melbourne Hospital, University of Melbourne

Parkville, VIC 3050 (Australia)

E-Mail bernard.yan@mh.org.au 
efit of neuroimaging to broaden treatment eligibility and the issues associated with general anesthesia will be discussed in this review. Key Messages: Questions remain over the applicability of mechanical thrombectomy to stroke subgroups including wake-up strokes and basilar artery thrombosis. The role of imaging is integral to this process and can lead to broadening eligibility criteria in the future. Workflow practices have been streamlined in the 5 positive randomized controlled studies, but guidelines will need to be revised accordingly if similar patient outcomes are to be replicated in a wider population.

(C) 2015 S. Karger AG, Base

\section{Introduction}

Acute stroke treatment has evolved significantly over the last 20 years. It was initially galvanized by the validation of intravenous recombinant tissue plasminogen activator (IV rt-PA) in studies demonstrating improved clinical outcomes in patients treated within $3 \mathrm{~h}$ of stroke ictus [1, 2]. Despite the clinical benefits of IV rt-PA, disappointments remained concerning modest recanalization rates, ranging between $4.4 \%$ for distal internal carotid artery (ICA) occlusion, $4 \%$ for basilar artery occlusions and 30\% for middle cerebral artery (MCA) M1 and M2 segment occlusions [3]. The importance of vascular recanalization in relation to outcome was established in earlier studies [4, 5], driving research efforts to develop new endovascular devices to increase recanalization rates. This was provisionally shown in the MERCI trial, which achieved $46-48 \%$ rates of revascularization in arterial occlusion resistant to IV rt-PA [6]. However, 3 subsequent trials - Endovascular Therapy after Intravenous t-PA versus t-PA Alone for Stroke (IMS III) [7], Endovascular Treatment for Acute Ischemic Stroke (SYNTHESIS Expansion) [8] and A Trial of Imaging Selection and Endovascular Treatment for Ischemic Stroke (MR RESCUE) [9] - failed to definitively demonstrate superiority of intra-arterial therapy. This review evaluates the recent evidence in support of mechanical thrombectomy (MET) for acute ischemic stroke with large artery occlusion.

\section{Brief Summary of the Five Positive Randomized Controlled Studies and Outline of the Key Concerns}

MR CLEAN Trial (Multicenter Randomized Clinical Trial of Endovascular Treatment for Acute Ischemic Stroke in the Netherlands)

MR CLEAN [10] measured the efficacy of new-generation stent retrievers Trevo Pro and Solitaire ${ }^{\mathrm{TM}} \mathrm{FR}$ in $81.5 \%$ of its interventional group and showed that $32.6 \%$ of the interventional group compared to $19.1 \%$ of the standard therapy group achieved a modified Rankin Scale (mRS) score of $0-2$ ( $\mathrm{p}<0.01)$, adjusted odds ratio (OR) 2.16 (1.39-3.38), numbers needed to be treated (NNTB) 8 . Mortality was $18.4 \%$ with no statistical difference when compared with its control, and consistent with other studies such as Endovascular Revascularization with Solitaire Device versus Best Medical Therapy in Anterior Circulation Stroke within $8 \mathrm{~h}$ (REVASCAT) and Endovascular Treatment for Small Core and Proximal Occlusion Ischemic Stroke (ESCAPE). Symptomatic intracranial hemorrhage (sICH) was 7.7\% for the interventional group with no statistical difference when compared to the control arm.

\section{ESCAPE Trial}

The ESCAPE [11] trial was ceased early after an unplanned interim analysis following the publication of the MR CLEAN study. ESCAPE used the Solitaire stent in $86.7 \%$ of its interventional cohort. It showed that $53 \%$ of the intervention group compared to $29.3 \%$ of the control 
group had a favorable outcome ( $\mathrm{mRS} \leq 2 ; \mathrm{p}<0.001$ ). There was an adjusted OR 3.1 [confidence interval (CI) 2.0-4.7] greater likelihood of achieving a 1-point mRS shift at 3 months if intervention was commenced ( $\mathrm{p}<0.001)$. NNTB was 4 with mortality 10.4 and $19.0 \%$ between the intervention and control arms $(p=0.04)$. sICH was $3.6 \%$ in the intervention group and was not statistically significant.

EXTEND IA Trial (Extending the Time for Thrombolysis in Emergency Neurological Deficits with Intra-Arterial Therapy)

The EXTEND IA [12] used Solitaire FR (flow restoration) in $77.1 \%$ of its cohort. The trial was ceased after randomization of 70 patients with the study showing that the interventional group achieved better functional outcome at 90 days than the alteplase-only group. Generalized OR was 2.0 (95\% CI 1.2-3.8; $\mathrm{p}=0.006$ ). The proportion of patients achieving an $\mathrm{mRS}$ score $\leq 2$ in the intervention group versus the control group was 71 versus $40 \%$, respectively [OR 4.2 (1.4-12); $\mathrm{p}=0.01$ ]. NNTB was 2.8 for a 1-point positive $\mathrm{mRS}$ shift. Mortality rates were $9 \%$ in the intervention group and not statistically significant. $0 \%$ of the intervention group reported sICH, but this may have been due to the small cohort before study termination.

SWIFT PRIME Trial (Solitaire with the Intention for Thrombectomy as Primary Treatment for Acute Ischemic Stroke)

SWIFT PRIME [13] used Solitaire FR and Solitaire 2 devices in $89 \%$ of their cohort. The study was ceased by the data and safety monitoring board after an interim review found a substantial benefit for patients treated in the intervention arm.

The study recruited 196 patients and measured IV t-PA and neurovascular thrombectomy against IV t-PA alone. The interventional group, compared to the control, achieved favorable outcomes (mRS 0-2) at 60 and 35\%, respectively [adjusted OR 2.03 (95\% CI 1.36-3.03); NNTB 4; $\mathrm{p}<0.001]$. An mRS shift was seen in 1 patient for every 2.6 patients treated. There was no statistical difference in $\operatorname{sICH}(p=0.50)$ or mortality $(p=0.12)$.

\section{REVASCAT Trial}

REVASCAT [14] was ceased early after the publications of ESCAPE, EXTEND IA and MR CLEAN resulted in a loss of equipoise rather than meeting any predetermined criteria for early cessation [14].

Intervention led to a 1-point mRS improvement to the adjusted OR 1.7 (95\% CI 1.05-2.8). When comparing the intervention group with the control group, the proportion of patients achieving independent functional outcome (mRS 0-2) was 43.7 versus $28.2 \%$ [adjusted OR 2.1 (95\% CI 1.1-4.0)]. NNTB was 6.5 to prevent disability or death. Revascularization TICI $2 b-3$ (thrombolysis in infarction score; $2 \mathrm{~b}=$ complete filling of all of the expected vascular territory is visualized, but the filling is slower than normal; $3=$ complete perfusion, antegrade flow into the bed distal to the obstruction occurs) was $65.7 \%$ in the intervention group. No statistically significant difference was found in mortality or sICH when comparing the two groups.

\section{Discussion}

\section{Advanced Age Is Not a Contraindication to MET}

Both age and National Institutes of Health Stroke Scale (NIHSS) score are independent variables impacting outcome for MET with both influencing treatment benefit [15]. However, in ESCAPE, patients $>80$ and $<80$ years old maintained a positive treatment benefit of OR 2.06 and 1.78, respectively, while MR CLEAN found patients $>80$ experienced an adjusted OR of 3.24 . 
Palaniswami and Yan: Mechanical Thrombectomy Is Now the Gold Standard for Acute

\section{Severe Stroke Patients Also Benefit from MET}

The median age range for patients was similar across the 5 studies (table 1). The SWIFT PRIME subgroup analysis stratified patients according to NIHSS $\leq 17$ and $>17$ and found that there was no difference in treatment effect ( $p$ interaction $=0.68$ ). Similarly, the ESCAPE trial stratified their subgroup into NIHSS $<6-19$ and $\geq 20$ and found no diminishment in treatment effect, whilst the MR CLEAN subgroup analysis found that the benefit of MET was greatest in those with NIHSS $\geq 20$ (OR 1.85). Amongst the 2015 publication series, the median NIHSS was 17-18 except for the EXTEND IA trial which recorded a median NIHSS of 13 in its control.

\section{Study Design and Selection Criteria}

The recent study series were all prospective trials focused on confirming occlusions in the proximal MCA and ICA through imaging. The use of newer generation stent retrievers (Solitaire FR, Solitaire 2 and Trevo Pro devices) was consistent amongst the 5 studies. The time frame for recruitment centered around 6-8 h, with ESCAPE recruiting 15.5\% of its cohort between 6 and $12 \mathrm{~h}$. The studies relied on computerized tomography (CT) angiography, magnetic resonance angiography (MRA) or digital subtraction angiography to confirm an occlusion, while EXTEND IA went further by using CT perfusion (CTP) to accept patients according to mismatch profile and ischemic core volume. MR CLEAN was deemed to have the most relaxed criteria as they did not utilize the Alberta Stroke Program Early Computed Tomography Score (ASPECTS) to exclude patients, whilst ESCAPE opted to use collateral circulation in its study design (table 2).

The protocol for MR CLEAN more realistically reflects current clinical capabilities and could form the basis of guidelines [16] with the option of including CTP parameters (as defined by EXTEND IA) when available. Potentially, 7-15\% of patients could benefit from intervention but treatment numbers are far less [17]. Broader criteria would encourage referrals for intervention and allow guidelines to be refined faster as data volume increases.

The inclusion criteria for REVASCAT and a portion of the MR CLEAN cohort encompassed patients with occlusions 30 min after intravenous alteplase treatment. REVASCAT boasted a higher proportion of its cohort achieving a favorable outcome $(43.7 \%)$ compared to MR CLEANS (32.6\%). It is more likely that the higher rates of favorable outcome reported in REVASCAT were due to the study's smaller cohort and exclusion of patients with large early ischemic changes (as defined by an ASPECTS >7). Using the failure of intravenous thrombolysis (IVT) to select eligible patients for treatment could be a valid strategy especially when access to imaging is restricted. It also opens the option of administering treatment en route to specialized stroke centers, though a previous analysis of the 'drip and ship method' has found patients to fare statistically worse [18].

REVASCAT was unique in using a government-based registry called SONIIA that automatically recruited patients when they fulfilled predefined criteria upon presentation and treatment. This was an externally validated database designed to effectively enroll patients for the REVASCAT study. Despite this, the registry recorded only $15.6 \%$ of all ischemic stroke admission in Catalonia and recruited $0.04 \%(n=103)$ for the study. A generalized registry may be appropriate in the future, but selecting patients so stringently in these formative stages would delay our ability to refine and practice protocols. The total number of endovascular procedures within the SONIIA registry was $20.9 \%$, indicating that we may see a similar percentage treated in the future after protocol amendments.

\section{Complications of Stent Retrievers}

MR CLEAN showed an increased risk of new ischemic strokes $(\mathrm{p}<0.001)$ in different territories, with $5.6 \%$ of patients versus $0.4 \%$ experiencing new ischemic strokes within 90 days for the intra-arterial group and standard therapy group, respectively, in addition to emboli- 

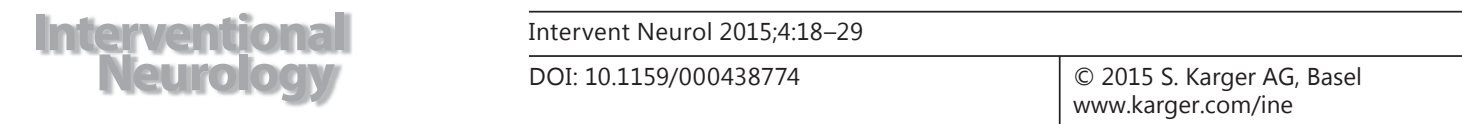

Palaniswami and Yan: Mechanical Thrombectomy Is Now the Gold Standard for Acute Ischemic Stroke: Implications for Routine Clinical Practice
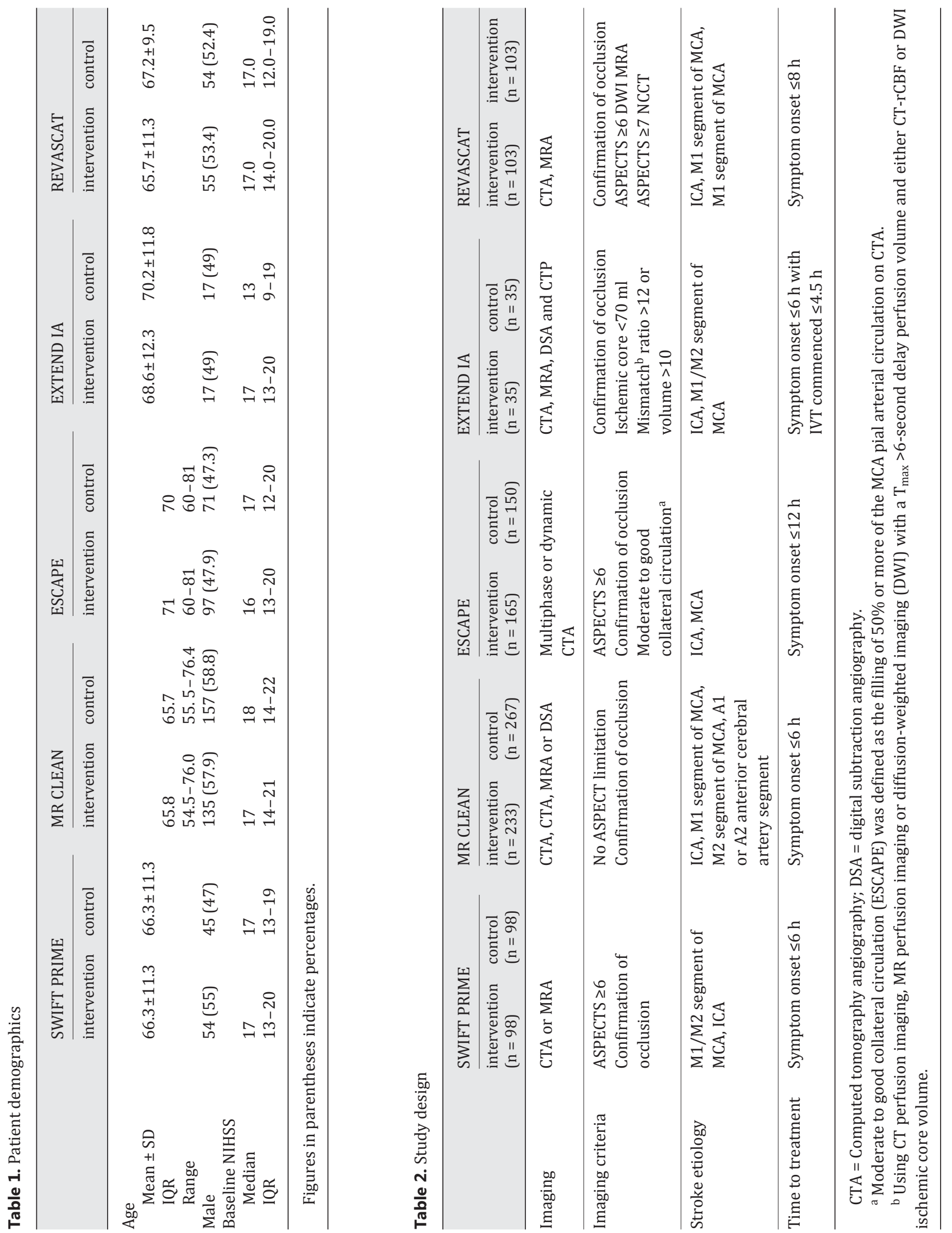
Table 3. Procedure-related events

\begin{tabular}{llllll}
\hline & SWIFT PRIME & MR CLEAN & ESCAPE & Extend IA & REVASCAT \\
\hline Device used & $\begin{array}{l}\text { Solitaire FR } \\
\text { (flow restoration) } \\
\text { Solitaire 2 device }\end{array}$ & $\begin{array}{l}\text { Trevo Pro } \\
\text { solitaire FR }\end{array}$ & $\begin{array}{l}\text { Solitaire } \\
\text { stent }\end{array}$ & $\begin{array}{l}\text { Solitaire FR } \\
\text { (flow restoration) }\end{array}$ & $\begin{array}{l}\text { Solitaire device } \\
\text { (solitaire FR) }\end{array}$ \\
& n.a. & & & \\
\hline Device used in trial & $89 \%$ & $81.5 \%$ & $86.7 \%$ & $77.1 \%$ & $95.1 \%$ \\
Embolization into another vessel territory & n.a. & $0.9 \%$ & 0.6 & $2.9 \%$ & $4.9 \%$ \\
Arterial perforation & n.a. & $1.7 \%$ & 0.6 & 4.9 & 3.9 \\
Arterial dissection & n.a. & n.a. & 7.2 & $2.9 \%$ & 10.7 \\
Access site hematoma & $37 \%$ & $37.8 \%$ & $9.1 \%$ & $36 \%$ & $6.8 \%$ \\
General anesthesia & & & & \\
\hline
\end{tabular}

n.a. $=$ Not available

zation rates of $8.6 \%$ in the intervention group. The device related or procedural complication rate was $10.9 \%$ in ESCAPE with recurrent strokes occurring in 4.9\%. EXTEND IA reported a $5.7 \%$ incidence of recurrent strokes. However, REVASCAT reported 1\% of cerebrovascular embolization, and SWIFT PRIME reported 0 adverse events. The lower rates of device complication reported in REVASCAT and SWIFT PRIME could reflect the benefit in entrusting the device with competent practitioners. REVASCAT required clinicians to have performed at least 20 MET procedures with the Solitaire device prior to their involvement in the study.

A retrospective analysis of MET (Solitaire FR and Penumbra 3D devices) in a high-volume neurocenter with $24 / 7$ stroke care found lower rates of recurrent strokes $(2 \%)$ and sICH (5\%), and observed a correlation between complications and prolonged groin puncture time $(>1 \mathrm{~h})$ to revascularization/end result [19]. ESCAPE recorded the lowest symptom onset to groin puncture time (median $185 \mathrm{~min}$ ) and recorded proportionally lower complication rates relative to the other studies (table 3 ).

The lower rates of sICH observed in the trial series could also be due to the stricter selection criteria followed in the recent study series. The lower rates of recurrent strokes may also be due to operator expertise garnered from operating in a specialized stroke center. REVASCAT recorded the highest percentage of arterial perforation (4.9\%) but also employed the highest percentage of stent retrievers in its trial (95.1\%).

\section{Workflow Parameter Improvements}

A post hoc analysis of the IMS III trial found that for every 30-min delay in reperfusion, there was a decreased likelihood of good clinical outcome by $12 \%$ (adjusted analysis; adjusted relative risk $0.88(0.80-0.98)]$ [20]. They reiterated the need to decrease door to needle time and cited a study where this was reduced to $20 \mathrm{~min}$ in a Finnish center, suggesting that ideal mean times to perfusion be $223 \mathrm{~min}$ [21]. When measuring workflow practices, the median time to reperfusion was 332 and 355 min in the studies of MR CLEAN and REVASCAT, respectively. This may suggest that improved technology and stricter selection criteria (compared to previous studies) had a greater bearing on outcome than time to reperfusion. Despite the longer times to reperfusion recorded in MR CLEAN, the study still observed a treatment effect of OR 2.16 (1.39-3.38).

Amongst both the 2015 and 2013 trials, the time to groin puncture from symptom onset was reported consistently and ranged from a median of 210-269 min in the 2015 trials to a median of 208-381 min in the 2013 trials. The use of imaging and IVT are significant components in the mean median time to treatment. A previous study found baseline imaging to groin 
puncture was delayed by $32 \mathrm{~min}$ (mean) when IVT was prescribed and 18 min (mean delay) when MRA was used. For every 1-hour delay in reperfusion, the odds of good clinical outcome decreased by 38\% [22] (table 4).

The 2015 trials employed measured to exceed recommended treating times. SWIFT PRIME for example targeted hospital presentation to groin puncture times at $70 \mathrm{~min}$ and achieved a median of $90 \mathrm{~min}$, which is significantly shorter than the $120 \mathrm{~min}$ recommended by many guidelines.

\section{Recanalization and Outcome}

A 2007 meta-analysis reviewing 53 studies $(n=2,066)$ that investigated recanalization rates found that good functional outcome ( $\mathrm{mRS} \leq 2)$ occurred (OR $=4.43 ; 95 \% \mathrm{CI} 3.32-5.91$ ), and the OR for the likelihood of mortality was 0.24 (95\% CI $0.16-0.35$ ) with no statistical difference in the incidence of sICH [23]. Furthermore, a post hoc analysis of IMS III found that recanalization was more frequent in patients with ICA and M1 occlusion pathology. With IVT alone, ICA recanalization can be an abysmal $4-8 \%$ with a favorable outcome achieved in $0-29 \%$ at 90 days [24].

MR CLEAN observed lower TICI $2 \mathrm{~b}-3$ recanalization, and at $58.7 \%$ it was below the range of $65.7-88 \%$ achieved in the other trials. Despite this, it observed a greater treatment benefit [adjusted OR 2.16 (1.39-3.38)] relative to REVASCAT adjusted OR [2.1 (1.1-4.0)] and SWIFTPRIME adjusted OR [2.03 (1.36-3.03)]. EXTEND IA found a correlation in their subanalysis between reperfusion and outcome, concluding that a $90 \%$ increased perfusion leads to OR 4.5 (95\% CI 2.2-9.0; $\mathrm{p}<0.001)$ improved functional outcome. The proportionally lower rates of recanalization (TICI $2 \mathrm{~b}-3$ ) in the MR CLEAN and REVASCAT studies (65.7\%) may be due to the inclusion of treatment-resistant patients (intervention offered only if intravenous alteplase could not establish reperfusion within $30 \mathrm{~min}$ ). Even so, the levels of recanalization achieved across the 5 studies were greater than in the controversial MR RESUCE (25\%) and ISM III (23-44\%) trials. The use of newer-generation stent retrievers may explain this as they exceeded $80 \%$ in all of the trials except EXTEND IA (77.1\%; table 5).

\section{Hub-and-Spoke Systems to Deliver MET}

A delay in treatment is inevitable when transferring patients, with one study estimating the median transfer time to be $104 \mathrm{~min}$ [25]. In the SWIFT PRIME study differences from symptom onset to groin puncture were 275 (IQR 245-334) and $179.5 \mathrm{~min}$ (IQR 147-238) when comparing patients initially treated by hospitals outside the study with those referred immediately to hospitals within the study.

It is unrealistic to expect every stroke center to specialize in neurointerventional surgery as only $4-14 \%$ of patients suffering ischemic strokes are eligible for neurovascular thrombectomy [26]. There is an argument to centralize care as it can improve workflow measures and thus benefit patients. Such measures could realistically be achieved through a hub-andspoke model while also providing the caseload for clinicians to practice MET [27]. A sample of hospitals from 2008 found that only $2.6 \%$ performed $\geq 10$ endovascular treatments for acute ischemic stroke with a trend favoring centralization [28]. Basing an MET model for resource distribution on how unruptured aneurysms are managed may be appropriate. With unruptured aneurysms, higher treatment numbers correlated with decreased morbidity and mortality for high-volume hospitals and practitioners [29].

\section{Advanced Neuroimaging as a Triage Tool}

A subgroup analysis in IMS III found that demonstrating an occlusion on baseline CT led to a higher likelihood of favorable outcome after endovascular therapy compared with when an occlusion was not confirmed (mRS 0-2, 47.2 vs. 38.5\%; $p=0.0114$ ) [30]. This has been a 


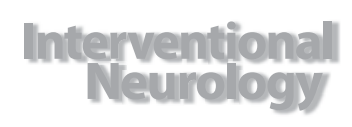

Intervent Neurol 2015;4:18-29

Palaniswami and Yan: Mechanical Thrombectomy Is Now the Gold Standard for Acute Ischemic Stroke: Implications for Routine Clinical Practice
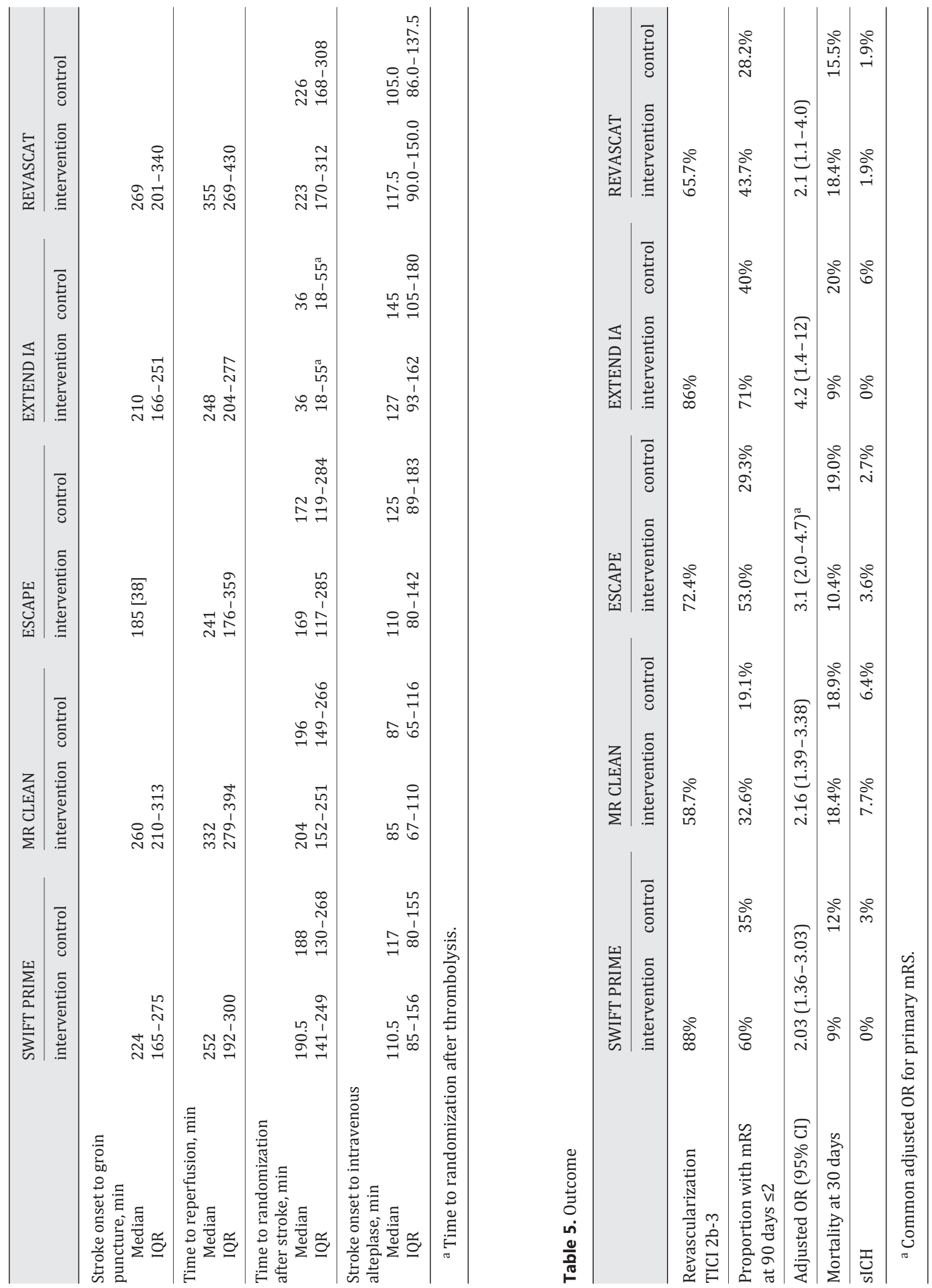
significant point of contention and could explain why recent studies investigating endovascular therapy have all adopted imaging to confirm the presence of an occlusion.

The decision to use ASPECTS was adopted by all the 5 studies except MR CLEAN. Despite this, $94.4 \%$ of the MR CLEAN cohort was recruited with scans above ASPECTS 5. A subgroup analysis of the study accordingly found the greatest benefit in this subgroup [10]. REVASCAT, SWIFT PRIME and ESCAPE found no statistically significant benefit of treating patients with thrombectomy when stratified by ASPECTS; however, these studies were not sufficiently powered for subgroup analysis and only recruited patients with ASPECTS $\geq 6$.

EXTEND IA and SWIFT PRIME used CTP to identify patients with appropriate image profiles dependent on their mismatch profile and ischemic core volume. The EXTEND trial used a mismatch ratio $>1.2$ and an ischemic core lesion volume of $<70 \mathrm{ml}$. SWIFT PRIME used either MRI or CT for a mismatch ratio of $\geq 1.8$ but limited its ischemic core to $<50 \mathrm{ml}$. Previous automated software in the MR RESCUE trial failed to provide a mismatch classification in $42 \%$ of cases, while the number was $12.4 \%$ using the RAPID software. By using standardized software (RAPID software) to identify eligible patients, they set an important precedent which could have implications on workflow practice. EXTEND IA achieved a median of 5 min $20 \mathrm{~s}$ across 20 trial sites for image processing and a gap of 6 min $28 \mathrm{~s}$ between noncontrast CT and CTP acquisition. The use of MRI caused a median time increase of $78 \mathrm{~min}$ when compared to CTP and may not provide any additional information that could inform treatment decisions [31]. ESCAPE took into account collateral circulation after a post hoc analysis of IMS III [32], with additional studies demonstrating a strong interaction between collateral circulation and revascularization [33]. Automating this process similar to the EXTEND IA and SWIFT PRIME trials may provide an intermediate solution for facilities without CTP capabilities.

The use of CTP to exclude patients based on neuroimaging may have also impacted on the incidence of sICH, with interventional arms of both SWIFT PRIME and EXTEND IA recording $0(0 \%)$ in their study. MR CLEAN by contrast reported a $7.7 \%$ incidence of sICH in its intervention group. There seems to be a positive trend correlating increasing imaging restrictions to better outcomes and decreased complications. However, the balance between increased patient selectivity and benefit of treatment must be undertaken.

\section{General Anesthesia and Sedation}

The use of general anesthesia (GA) over sedation when performing MET is still under discussion. However, amongst the studies published in 2015, varying levels of general anesthetics were used. The trials of REVASCAT and ESCAPE reported the use of GA in 6.8 and 9.1\% of its patients, respectively, while the remaining 3, MR CLEAN, EXTEND IA and SWIFT PRIME reported a 36-38\% use of GA in their interventional arm. Despite the substantial differences in GA use, the treatment benefit for neurovascular intervention was fairly consistent amongst the 5 studies. A post hoc analysis of the MR CLEAN study into the use of GA found similar proportions of death at 30 days for 18 and 19\% for GA and non-GA, respectively. The rates for sICH were $8 \%$ for both groups. They did however find a treatment effect on mRS (0-2) outcome between non-GA [OR 2.13 (95\% CI 1.70-4.59)] and GA [OR 1.09 (95\% CI 0.562.12)]. A 2014 meta-analysis $(n=1,956)$ assessing the interaction of GA compared with sedation in endovascular therapy found there was a higher chance of death [OR $=2.59(95 \%$ CI 1.87-3.58)], increased likelihood of respiratory complications and a lower chance of good functional outcome [mrS 0-2; OR $=0.43$ (95\% CI 0.35-0.53)]. There was no statistical difference in procedural time $(\mathrm{p}=0.28)[34]$.

\section{Future Direction}

The patient selection in these trials has an implication on how MET in clinical practice proceeds. The trials published in 2015 refer to proximal arterial occlusions quite specifically 
with adjuvants such as collateral circulation and ischemic core volumes, further refining the patients deemed eligible for intervention. Ongoing trials such as the SITS Open, which broadens patient recruitment to include patients with posterior circulation occlusions including basilar artery occlusions, are necessary to add to this growing body of evidence [35].

The infrastructure required to accommodate MET is extensive and undoubtedly expensive. The technology is still developing, and the recent trials were conducted in highly idealized circumstances (e.g. time to intervention, coordination of teams etc). Broadening the criteria will only help if policy, coordination and resources are scaled appropriately [36]. For example, $22 \%$ of eligible patients were excluded from the EXTEND IA trial due to clinician unavailability, and in such a scenario a more restrictive criteria (as seen in EXTEND IA) may be more appropriate [12].

Investigations must also be made into improving standardized software to identify suitable patients for intervention. Countless studies have shown a connection between imaging parameters and outcome in ischemic stroke. The use of RAPID software in SWIFT PRIME and EXTEND-IA heralds the first steps in standardizing eligibility protocol and will undoubtedly lead to the practice being more common in the future.

The recent study series neglects patient subgroups categorized under wake-up strokes or those outside the treatment time frame. Studies such as Perfusion Imaging Selection of Ischemic Stroke Patients for Endovascular Therapy (POSITIVE) and Trevo and Medical Management versus Medical Management Alone in Wake Up and Late Presenting Strokes (DAWN) and Study of Intravenous Thrombolysis with Alteplase in MRI-Selected Patients (MR WITNESS) are currently underway and will provide crucial data to validate this hypothesis [37].

\section{Conclusion}

Despite variable differences in intervention times and revascularization rates, the treatment effect of MET as part of endovascular therapy seems to be consistent amongst the 5 published trials in 2015. The impact of imaging seems to be significant as all 5 trials employed measured to confirm occlusions, while trials such as EXTEND IA went even further and utilized CT perfusion in their patient cohort. There seems to be mounting evidence against the use of GA, and future trials may investigate this issue more thoroughly.

Questions still remain whether transferring patients to specialized stroke centers using a hub-and-spoke model is the best way to allocate resources. Without imaging, it is difficult to justify referring patients onwards as only a very defined subgroup of ischemic stroke patients have shown to benefit from intervention in these recent trials.

Studies are usually conducted under idealized circumstances, but this means that it is also harder to generalize their findings. All of the recent studies discussed in this review were conducted in specialized endovascular centers, which enabled them to integrate novel workflow designs. It will take time before such workflow practices become more widespread, and outcomes may not mirror the levels observed in this study series until protocols become standardized.

\section{Disclosure Statement}

The authors declare that there is no conflict of interest in writing this article. Assoc. Prof. Bernard Yan was a lead investigator in the EXTEND IA trial that was subject to analysis in this article. 


\begin{tabular}{l|l}
\hline Intervent Neurol 2015;4:18-29 \\
\hline DOI: 10.1159/000438774 & $\begin{array}{l}\text { C 2015 S. Karger AG, Basel } \\
\text { www.karger.com/ine }\end{array}$ \\
\hline
\end{tabular}

Palaniswami and Yan: Mechanical Thrombectomy Is Now the Gold Standard for Acute Ischemic Stroke: Implications for Routine Clinical Practice

\section{References}

1 Tissue plasminogen activator for acute ischemic stroke. The National Institute of Neurological Disorders and Stroke rt-PA Stroke Study Group. N Engl J Med 1995;333:1581-1587.

-2 Hacke W, Kaste M, Fieschi C, et al: Intravenous thrombolysis with recombinant tissue plasminogen activator for acute hemispheric stroke. The European Cooperative Acute Stroke Study (ECASS). JAMA 1995;274:1017-1025.

-3 Bhatia R, Hill MD, Shobha N, et al: Low rates of acute recanalization with intravenous recombinant tissue plasminogen activator in ischemic stroke: real-world experience and a call for action. Stroke 2010;41:2254-2258.

$\checkmark 4$ Mori E, Yoneda Y, Tabuchi M, et al: Intravenous recombinant tissue plasminogen activator in acute carotid artery territory stroke. Neurology 1992;42:976-982.

5 Yamaguchi T, Hayakawa T, Kiuchi H: Intravenous tissue plasminogen activator ameliorates the outcome of hyperacute embolic stroke. Cerebrovasc Dis 1993;3:269-272.

6 Smith WS, Sung G, Starkman S, et al: Safety and efficacy of mechanical embolectomy in acute ischemic stroke: results of the MERCI trial. Stroke 2005;36:1432-1438.

7 Broderick JP, Palesch YY, Demchuk AM, et al: Endovascular therapy after intravenous t-PA versus t-PA alone for stroke. N Engl J Med 2013;368:893-903.

8 Ciccone A, Valvassori L, Nichelatti M, et al: Endovascular treatment for acute ischemic stroke. N Engl J Med 2013;368:904-913.

-9 Kidwell CS, Jahan R, Gornbein J, et al: A trial of imaging selection and endovascular treatment for ischemic stroke. N Engl J Med 2013;368:914-923.

10 Berkhemer OA, Fransen PS, Beumer D, et al: A randomized trial of intraarterial treatment for acute ischemic stroke. N Engl J Med 2015;372:11-20.

-11 Goyal M, Demchuk AM, Menon BK, et al: Randomized assessment of rapid endovascular treatment of ischemic stroke. N Engl J Med 2015;372:1019-1030.

$\checkmark 12$ Campbell BC, Mitchell PJ, Kleinig TJ, et al: Endovascular therapy for ischemic stroke with perfusion-imaging selection. N Engl J Med 2015;372:1009-1018.

-13 Saver JL, Goyal M, Bonafe A, et al: Stent-retriever thrombectomy after intravenous t-PA vs. t-PA alone in stroke. N Engl J Med 2015;372:2285-2295.

14 Jovin TG, Bonafe A, Cobo E, et al: Thrombectomy within 8 hours after symptom onset in ischemic stroke. N Engl J Med 2015;372:2296-2306.

-15 Almekhlafi MA, Davalos A, Bonafe A, et al: Impact of age and baseline NIHSS scores on clinical outcomes in the mechanical thrombectomy using solitaire FR in acute ischemic stroke study. AJNR Am J Neuroradiol 2014;35: 1337-1340.

16 Fransen PS, Beumer D, Berkhemer OA, et al: MR CLEAN, a multicenter randomized clinical trial of endovascular treatment for acute ischemic stroke in the Netherlands: study protocol for a randomized controlled trial. Trials 2014;15:343.

17 Adamczyk P, Attenello F, Wen G, et al: Mechanical thrombectomy in acute stroke: utilization variances and impact of procedural volume on inpatient mortality. J Stroke Cerebrovasc Dis 2013;22:1263-1269.

18 Sheth KN, Smith EE, Grau-Sepulveda MV, et al: Drip and ship thrombolytic therapy for acute ischemic stroke: use, temporal trends, and outcomes. Stroke 2015;46:732-739.

19 Behme D, Gondecki L, Fiethen S, et al: Complications of mechanical thrombectomy for acute ischemic stroke - a retrospective single-center study of 176 consecutive cases. Neuroradiology 2014;56:467-476.

20 Khatri P, Yeatts SD, Mazighi M, et al: Time to angiographic reperfusion and clinical outcome after acute ischaemic stroke: an analysis of data from the Interventional Management of Stroke (IMS III) phase 3 trial. Lancet Neurol 2014;13:567-574.

-21 Meretoja A, Strbian D, Mustanoja S, et al: Reducing in-hospital delay to 20 minutes in stroke thrombolysis. Neurology 2012;79:306-313.

22 Menon BK, Almekhlafi MA, Pereira VM, et al: Optimal workflow and process-based performance measures for endovascular therapy in acute ischemic stroke: analysis of the Solitaire FR thrombectomy for acute revascularization study. Stroke 2014;45:2024-2029.

23 Rha JH, Saver JL: The impact of recanalization on ischemic stroke outcome: a meta-analysis. Stroke 2007;38: 967-973.

24 Demchuk AM, Goyal M, Yeatts SD, et al: Recanalization and clinical outcome of occlusion sites at baseline CT angiography in the Interventional Management of Stroke III trial. Radiology 2014;273:202-210.

25 Prabhakaran S, Ward E, John S, et al: Transfer delay is a major factor limiting the use of intra-arterial treatment in acute ischemic stroke. Stroke 2011;42:1626-1630.

26 Zaidat 00, Lazzaro M, McGinley E, et al: Demand-supply of neurointerventionalists for endovascular ischemic stroke therapy. Neurology 2012;79(suppl 1):S35-S41.

-27 Moynihan B, Davis D, Pereira A, et al: Delivering regional thrombolysis via a hub-and-spoke model. J R Soc Med 2010;103:363-369.

28 Grigoryan M, Chaudhry SA, Hassan AE, et al: Neurointerventional procedural volume per hospital in United States: implications for comprehensive stroke center designation. Stroke 2012;43:1309-1314.

-29 Brinjikji W, Rabinstein AA, Lanzino G, et al: Patient outcomes are better for unruptured cerebral aneurysms treated at centers that preferentially treat with endovascular coiling: a study of the national inpatient sample 2001-2007. AJNR Am J Neuroradiol 2011;32:1065-1070. 
30 Thomalla G: 'Time clock' or 'tissue clock'? The role of imaging in stroke management decisions. International Stroke Conference, Honolulu, February 2013. http://www.strokeforum.com/content/dam/internet/pm/ strokeforum/com_EN/documents/03_EStC2014_Thomalla.pdf.

31 Campbell BC, Yassi N, Ma H, et al: Imaging selection in ischemic stroke: feasibility of automated CT-perfusion analysis. Int J Stroke 2015;10:51-54.

-32 Menon BK, Qazi E, Nambiar V, et al: Differential effect of baseline CTA collateral status on clinical outcomes in patients enrolled in the IMS-3 trial. Stroke 2014;45(suppl 1):A7.

-33 Nambiar V, Sohn SI, Almekhlafi MA, et al: CTA collateral status and response to recanalization in patients with acute ischemic stroke. AJNR Am J Neuroradiol 2014;35:884-889.

34 Brinjikji W, Sohn SI, Almekhlafi MA, et al: Conscious sedation versus general anesthesia during endovascular acute ischemic stroke treatment: a systematic review and meta-analysis. AJNR Am J Neuroradiol 2015;36: 525-529.

35 https://sitsinternational.org/sits-projects/copy_of_sits-east.

-36 Adamczyk P, Attenello F, Wen G, et al: Mechanical thrombectomy in acute stroke: utilization variances and impact of procedural volume on inpatient mortality. J Stroke Cerebrovasc Dis 2013;22:1263-1269.

-37 Menon BK, Campbell BC, Levi C, et al: Role of imaging in current acute ischemic stroke workflow for endovascular therapy. Stroke 2015;46:1453-1461.

-38 DeSousa KG, Potts MB, Raz E, Nossek E, Riina HA: Turning point of acute stroke therapy: mechanical thrombectomy as a standard of care. World Neurosurg 2015;83:953-956. 